\title{
GEIDST
}

\section{INFECÇÃO POR NEISSERIA GONORRHOEAE NA CONSULTA DE IST DO HOSPITAL DE CURRY CABRAL - ANÁLISE RETROSPECTIVA DE 8 ANOS (2006-2013)}

\author{
Pedro Mendes-Bastos', Helena Toda Brito², Isaura Rodrigues³, Teresa Pina ${ }^{3}$, Cândida Fernandes ${ }^{4}$, Ana Rodrigues ${ }^{4}$, \\ Jorge Cardoso ${ }^{5}$ \\ IInterno de Dermatovenereologia/Resident of Dermatology and Venereology, Serviço de Dermatologia e \\ Venereologia, Hospital de Curry Cabral, Centro Hospitalar de Lisboa Central, Lisboa, Portugal \\ 2Interna de Dermatovenereologia/Resident of Dermatology and Venereology, Serviço de Dermatologia e \\ Venereologia, Hospital Distrital de Santarém, Santarém, Portugal \\ ${ }^{3}$ Assistente Hospitalar Graduada de Patologia Clínica/Graduated Consultant of Clinical Pathology, Serviço de \\ Patologia Clínica, Hospital de Curry Cabral, Centro Hospitalar de Lisboa Central, Lisboa, Portugal \\ ${ }^{4}$ Assistente Hospitalar Graduada de Dermatovenereologia/ Graduated Consultant of Dermatology and Venereology, \\ Serviço de Dermatologia e Venereologia, Hospital de Curry Cabral, Centro Hospitalar de Lisboa Central, Lisboa, \\ Portugal \\ ${ }^{5}$ Chefe de Serviço, Director/Consultant Chief and Head of Dermatology and Venereology, Serviço de Dermatologia e \\ Venereologia, Hospital de Curry Cabral, Centro Hospitalar de Lisboa Central, Lisboa, Portugal
}

RESUMO - Introdução: Assistiu-se nas últimas décadas a um aumento do número de casos de gonorreia notificados na Europa. Material e métodos: Foram revistos de forma retrospectiva os processos clínicos dos doentes observados na Consulta de IST do Hospital de Curry Cabral durante o período compreendido entre Janeiro de 2006 e Dezembro de 2013 (8 anos). O rastreio para a infecção por Neisseria gonorrhoeae é realizado em todos os doentes de forma sistemática através de técnica de diagnóstico molecular (BD Probetec-SDA system). Os autores procederam à caracterização epidemiológica e sociodemográfica bem como determinação da frequência da infecção por $N$. gonorrhoeae e respectivas localizações. Resultados: Durante o período em análise, num universo de 1735 doentes, foi diagnosticada gonorreia em 114 casos (6,6\%), 104 em homens e 10 em mulheres, com uma mediana de idades de 29 anos. $O$ diagnóstico de gonorreia verificou-se em 63 homens que têm sexo com homens (HSH) e 41 homens que têm sexo com mulheres. Dos doentes com diagnóstico de gonorreia, 26 eram seropositivos para o VIH (23\%). A localização uro-genital foi a mais frequente, com 103 casos, seguindo-se a infecção do recto em 12 doentes e infecção da orofaringe em 9 doentes. De todos estes diagnósticos, 17\% ocorreram em localizações nas quais os doentes não referiram sintomas (13 homens e 5 mulheres). Conclusões: Os resultados deste trabalho foram semelhantes aos dados publicados recentemente na literatura. Salienta-se o elevado número de casos em HSH. Neste contexto epidemiológico, a identificação deste agente em localizações sem queixas não é negligenciável, realçando a importância do rastreio extragenital em doentes assintomáticos nessas localizações.

PALAVRAS-CHAVE - Neisseria gonorrhoeae; Gonorreia; Doenças sexualmente transmissíveis.

\section{NEISSERIA GONORRHOEAE INFECTION IN A STD CLINIC, HOSPITAL DE CURRY CABRAL - 8 YEARS RETROSPECTIVE STUDY (2006-2013)}

ABSTRACT - Introduction: There has been in the last decades an increasing number of reported gonorrhea cases in Europe. Methods: We retrospectively reviewed the clinical records of patients attending Hospital de Curry Cabral's STD Clinic between January 2006 and December 2013 (8 years). The screening for Neisseria gonorrhoeae infection is per- 
formed systematically in all patients (molecular diagnosis technique - BD Probetec-SDA system). The authors assessed epidemiological and socio-demographic data and determined the frequency of N. gonorrhoeae infection. Results: During the reviewed period, out of 1735 patients, gonorrhea was diagnosed in 114 cases (6.6\%), 104 men and 10 women, with a median age of 29 years. The diagnosis of gonorrhea was found in 63 men who have sex with men (MSM) and 41 men who have sex with women. Of the totality of patients diagnosed with gonorrhea, 26 were HIV positive (24\%). The urogenital site was the most frequent with 103 cases, followed by anorectal in 12 patients and oropharyngeal in 9 patients. Of all these diagnoses, 17\% occurred in anatomic sites where patients did not report symptoms (13 men and 5 women). Conclusions: The results of this study were similar to data recently published in the literature. The high number of gonorrhea cases in MSM must be highlighted. In this epidemiological context, the identification of this agent in asymptomatic anatomic sites is not negligible, supporting the importance of extragenital screening.

KEY WORDS - Neisseria gonorrhoeae; Gonorrhea; Sexually transmitted diseases.
Conflitos de interesse: Os autores declaram não possuir conflitos de interesse.
No conflicts of interest.
Suporte financeiro: $O$ presente trabalho não foi suportado por nenhum subsídio ou bolsa.
No sponsorship or scholarship granted.
Direito à privacidade e consentimento escrito / Privacy policy and informed consent: Os autores declaram que pediram consentimento ao doente para usar as imagens no artigo. The authors declare that the patient gave written informed consent for the use of its photos in this article.
Aprovação Ética/Ethics Approval: Estudo de acordo com as Recomendações de Helsínquia e aprovado pela Comissão de Ética Hospitalar; Study in accordance with the amended Declaration of Helsinki and approved by the Ethics Committee.

Por decisão dos autores, este artigo não foi redigido de acordo com os termos do novo Acordo Ortográfico. Recebido/Received - Fevereiro/February 2015; Aceite/Accepted - Abril/April 2015

\section{Dr. Pedro Mendes Bastos}

Serviço de Dermatologia e Venereologia

Hospital de Curry Cabral

Rua da Beneficiência, n. 98

1069-166 Lisboa, Portugal

Email: pmendesbastos@gmail.com

\section{INTRODUÇÃO}

A gonorreia é uma infecção sexualmente transmissível (IST) causada pela bactéria Gram-negativa Neisseria gonorrhoeae. Segundo os dados epidemiológicos mais recentes, é a segunda IST bacteriana mais frequente na Europa. A N. gonorrhoeae tem uma afinidade natural para a mucosa do tracto genito-urinário inferior originando mais frequentemente infecção gonocóccica uro-genital. Outras possíveis portas de entrada deste agente são as mucosas oro-faríngea, ano-rectal e conjuntival. Embora raras, as suas complicações incluem, nas mulheres, doença inflamatória pélvica, gravidez ectópica e infertilidade e, nos homens, epididimite. ' Estima-se que a gonococcémia disseminada acompanhe $1-3 \%$ das infecções mucosas. ${ }^{2}$
Desde a viragem do milénio que se assistiu a um aumento dos casos notificados de gonorreia em vários países europeus, particularmente em populações consideradas de maior risco como os homens que têm sexo com homens ( $\mathrm{HSH}$ ) e os heterossexuais jovens de ambos os sexos. ${ }^{3-9}$

Este aumento pode dever-se a vários factores como rastreios mais frequentes, utilização de métodos diagnósticos mais sensíveis (particularmente técnicas de amplificação de ácidos nucleicos), aumento dos comportamentos de risco em certas subpopulações ou simplesmente optimização das notificações. ${ }^{3}$ Em Portugal foram notificados 442 casos de infecção gonocóccica no período compreendido entre 2009 e 2012, sendo a grande maioria das notificações oriundas da região de Lisboa e Vale do Tejo. 10 De acordo com as actuais 
Guidelines Europeias para o diagnóstico e tratamento da gonorreia em adultos ${ }^{11}$, o esquema terapêutico de 1. I linha para o tratamento da infecção gonocóccica aguda não complicada é a associação de ceftriaxone $500 \mathrm{mg}$ por via intramuscular e azitromicina $2 \mathrm{~g}$ por via oral em toma única. Definir estratégias para controlar o surgimento de gonorreia multi-resistente é actualmente um dos maiores desafios no campo das IST. Em Portugal, já foram reportados casos de N. gonorrhoeae resistentes à azitromicina mas não às cefalosporinas, embora já tenham sido detectadas estirpes com diminuição da sensibilidade a esta classe. ${ }^{12}$

\section{OBJECTIVOS}

Foi realizado um estudo retrospectivo baseado na análise dos processos clínicos da população observada na Consulta de IST do Hospital de Curry Cabral (HCC) no período compreendido entre Janeiro de 2006 e Dezembro de 2013 (8 anos). Os autores pretendem avaliar a prevalência da infecção por $N$. gonorrhoeae nesta população e proceder a uma caracterização sócio-demográfica e clínico-epidemiológica. Por último, interpretam-se os resultados no contexto nacional, comparando-os com outros estudos semelhantes realizados recentemente em Portugal.

\section{MATERIAIS E MÉTODOS}

A pesquisa de $N$. gonorrhoeae é efectuada sistematicamente em amostras de urina a todos os doentes observados na Consulta de IST do HCC por Ténica de Amplificação de Ácidos Nucleicos (TAAN). É utilizada a tecnologia "Strand Displacement Amplification Assay" (SDA) em Real-Time da Becton.Dickinson - Probe Tec ${ }^{T M}$ $E T$. System, que detecta qualitativamente a Neisseria gonorrhoeae após amplificação do alvo "Gene Pilin" do genoma desta bactéria. Para além disso, esta pesquisa é realizada em amostras de exsudado uretral, cervical, oro-faríngeo e ano-rectal quando justificado clínica ou epidemiologicamente. Desde 2013 que a pesquisa de $N$. gonorrhoeae é realizada de forma sistemática a todos os HSH nas 3 localizações pelo menos uma vez por ano, ou mais frequentemente caso se justifique por critérios clínicos ou epidemiológicos. São considerados critérios para definição de caso de infecção gonocóccica a positividade da pesquisa de $\mathrm{N}$. gonorrhoeae por técnica de diagnóstico molecular SDA em amostra de urina ou amostra de outro produto biológico, nomeadamente exsudado uretral ou exsudado cervical ou exsudado oro-faríngeo ou exsudado ano-rectal.

$\mathrm{Na}$ análise retrospectiva dos processos foram analisadas as seguintes variáveis: género, idade, país de origem, orientação sexual, motivo de consulta, presença de co-infecção com Chlamydia trachomatis e seropositividade para o vírus da imunodeficiência humana $(\mathrm{VIH})$.

\section{RESULTADOS}

Durante o período compreendido entre Janeiro de 2006 e Dezembro de 2013 (8 anos) foram observados 1735 indivíduos na consulta de IST do HCC, 1336 homens (77\%) e 399 mulheres (23\%). Destes 1735 indivíduos, todos rastreados, foram diagnosticados 114 casos de gonorreia (prevalência de 6,6\%). Dos 114 casos, 104 ocorreram em homens $(91,2 \%)$ e 10 ocorreram em mulheres $(8,8 \%)$, com uma mediana de idades de 29 anos. A Fig. 1 ilustra a distribuição por género e por faixa etária dos indivíduos diagnosticados no período do estudo. De acordo com o esperado, a maioria dos casos concentram-se na 3. ${ }^{\mathrm{a}}$ e $4 .^{\mathrm{a}}$ décadas de vida. A Fig. 2 permite avaliar o número de casos diagnosticados em cada ano. À semelhança de outros estudos portugueses, ${ }^{13,14}$ verificou-se um aumento do número de casos até ao ano de 2009. Em 2013 verificou-se uma subida significativa do número de casos diagnosticados (29 casos).

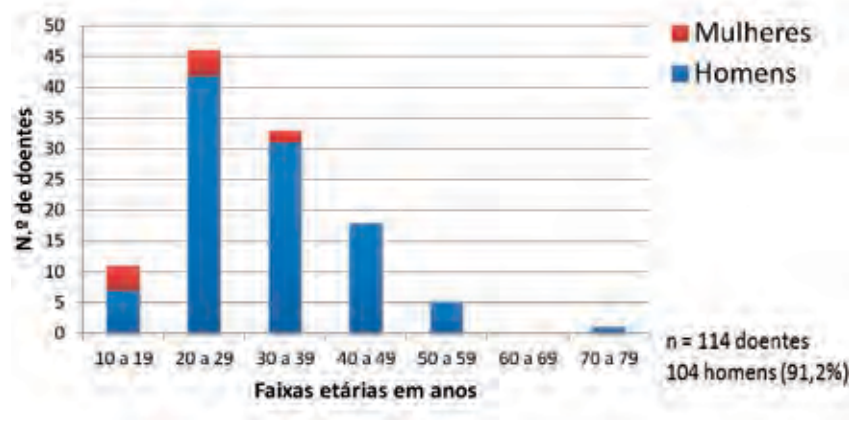

Fig 1 - Distribuição por género e por faixa etária dos diagnósticos de gonorreia na Consulta de IST do HCC de 2006 2013.

Quanto ao país de origem, destacam-se $81 \%$ dos casos com naturalidade portuguesa e $13 \%$ dos casos naturais dos PALOP e Brasil. Relativamente à orientação sexual, $55 \%$ dos casos (63 casos) ocorreram em $\mathrm{HSH}, 36 \%$ (41 casos) em homens que têm sexo com 


\section{GEIDST}

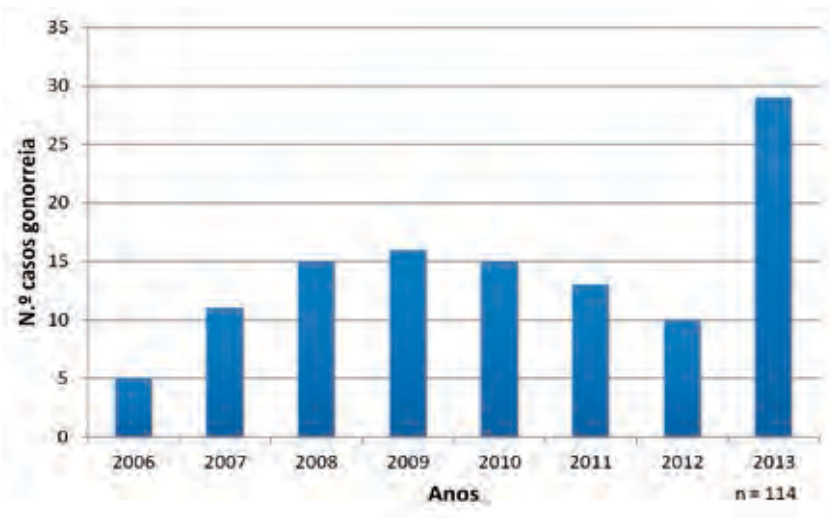

Fig 2 - Número de casos de gonorreia por ano na Consulta de IST do HCC de 2006-2013.

mulheres (HSM) e 9\% (10 casos) em mulheres que têm sexo com homens.

A Tabela 1 explicita qual o motivo de ida à Consulta de IST do HCC dos casos de gonorreia diagnosticados no período em análise. Destaca-se que $50 \%$ das mulheres diagnosticadas com gonorreia (5 casos) vieram à consulta como contactantes, estando totalmente assintomáticas no momento da consulta; a maioria (83\%) dos homens recorreu à consulta por exsudado uretral e $7 \%$ por exsudado anal. $O$ diagnóstico nas mulheres foi realizado após PCR $N$. gonorrhoeae positiva na urina $(8 / 10)$ e no exsudado cervical $(2 / 10)$. Quanto aos homens, todos foram testados em localização uro-genital (104 indivíduos), 13 foram testados em localização oro-faríngea e 18 em localização ano-rectal (vide Tabela 2). Quanto às positividades extragenitais em homens, verificaram-se $9 / 13$ casos de infecção gonocóccica oro-faríngea e 12/18 casos de infecção gonocóccica ano-rectal. Na Tabela 2, pode
Tabela 2 - Localizações anatómicas testadas por PCR N. gonorrhoeae em homens e respectivas positividades na Consulta de IST do HCC no período de 2006-2013.

\begin{tabular}{|l|c|c|c|}
\hline $\begin{array}{l}\text { LOCALIZAÇÕES } \\
\text { ANATÓMICAS } \\
\text { (HOMENS) }\end{array}$ & $\begin{array}{c}\text { URO- } \\
\text {-GENITAL }\end{array}$ & $\begin{array}{c}\text { ORO- } \\
\text {-FARÍNGEA }\end{array}$ & $\begin{array}{c}\text { ANO- } \\
\text {-RECTAL }\end{array}$ \\
\hline $\begin{array}{l}\text { Testadas } \\
\text { Positivas }\end{array}$ & 104 & 13 & 18 \\
\hline $\begin{array}{l}\text { Positivas } \\
\text { assintomáticas }\end{array}$ & 4 & 9 & 12 \\
\hline
\end{tabular}

ainda apreciar-se uma taxa de positividades $>50 \%$ em localizações extragenitais nas quais os indíviduos estavam assintomáticas no momento da consulta (5/9 orofaríngea e 6/12 ano-rectal). Realça-se que todos os homens testados em localizações extragenitais eram $\mathrm{HSH}$ e que apenas a partir de 2013 se começou o rastreio nas três localizações anatómicas a todos os HSH consultados. Globalmente, reunindo dados de homens e mulheres, $84 \%$ dos diagnósticos de gonorreia no período em estudo foram realizados na presença de sintomas sugestivos e $16 \%$ de todos os casos corresponderam a localizações assintomáticas no momento da Consulta de IST (Fig. 3).

A taxa global de co-infecção pela $N$. gonorrhoeae e pela $C$. trachomatis na população observada nesta consulta e no período em análise foi de $21 \%$, o que significa que 24 dos 114 casos de gonorreia ocorreram em doentes simultaneamente infectados por clamídia. A co-infecção pela N. gonorrhoeae e pela C. trachomatis assumiu uma maior preponderância no sexo feminino, com 7/10 mulheres infectadas simultaneamente contra 17/104 homens. Quanto à seropositividade para o $\mathrm{VIH}, 21$ dos 114 casos já tinham o

Tabela 1 - Motivos de ida à Consulta de IST do HCC dos casos de gonorreia diagnosticados no período de 2006-2013.

\begin{tabular}{|c|c|c|c|}
\hline SEXO & MOTIVO DE CONSULTA & NÚMERO DE DOENTES & TOTAL DE CASOS POR SEXO \\
\hline \multirow{3}{*}{ Masculino } & Exsudado uretral & 80 & \multirow{3}{*}{104} \\
\hline & Contactante assintomático & 1 & \\
\hline & Outros & 16 & \\
\hline \multirow[t]{2}{*}{ Feminino } & Disúria & 3 & \multirow[t]{2}{*}{10} \\
\hline & Contactante assintomático & 5 & \\
\hline
\end{tabular}




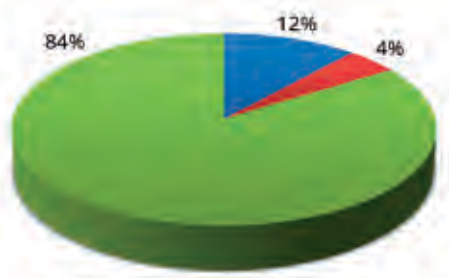

E Homens - Diagnósticos não referidos nas queixas

a Mulheres-Diagnósticos não referidos nas queixas w Diagnósticos sintomáticos

n diagnósticos assintomáticos $=18(16 \%)$ Hamens = 13/104; Mulheres = \$/10 $\mathrm{n}$ total diagnósticos $=114$

Fig 3 - Diagnósticos de gonorreia "sintomáticos" versus "assintomáticos" na Consulta de IST do HCC no período de 2006-2013.

diagnóstico de $\mathrm{VIH}$ prévio ao diagnóstico de gonorreia e outros 5 casos receberam o diagnóstico de novo de infecção pelo VIH concomitantemente; na globalidade, estes 26 doentes seropositivos para o $\mathrm{VIH}$ correspondem a $23 \%$ dos casos. Todos os doentes seropositivos para $\circ \mathrm{VIH}$ diagnosticados com gonorreia eram do sexo masculino.

\section{DISCUSSÃO E CONCLUSÕES}

Os dados epidemiológicos do ECDC (European Center for Disease Control) publicados mais recentemente (até 2011) apontam para um número crescente de casos notificados de gonorreia na Europa, com um pico de 38.779 casos em 2011 . Em Portugal, o número de casos notificados tem também crescido, com um pico de 120 notificações também em 2011 . $^{3}$

Os dados apresentados no presente estudo apontam para uma prevalência elevada $(6,6 \%)$ de infecção por N. gonorrhoeae na população observada na Consulta de IST do Hospital de Curry Cabral. As limitações inerentes a uma amostra de doentes que recorreram a uma Consulta de IST não permitem a generalização dos dados obtidos para a população portuguesa geral, mas permitem a avaliação das tendências da incidência desta infeção na população. De acordo com o esperado, a mediana de idades dos doentes foi de 29 anos, concentrando-se a maioria dos casos nas 3. e 4. ${ }^{\mathrm{a}}$ décadas de vida. Ao comparar estes dados com outros estudos nacionais recentes ${ }^{13,14}$ constata-se que a prevalência de gonorreia nesta consulta é superior à de outras séries (Tabela 3). Em 2013 verificou-se uma subida significativa do $\mathrm{n}$. - de casos diagnosticados (29 casos), em possível relação com o início de rastreio nas 3 localizações em HSH. Apesar de 13\% dos doentes infectados serem naturais dos PALOP ou Brasil, a infecção foi provavelmente contraída em Portugal. A preponderância desta infecção no sexo masculino (aproximadamente 9 homens para 1 mulher) reflecte a maior afluência de homens a esta consulta, salientando-se também que $60,6 \%$ dos casos no sexo masculino eram HSH. De facto, verificou-se um número relevante

Tabela 3 - Comparação de resultados do presente estudo sobre infecção por N. gonorrhoeae (Consulta de IST do HCC no período de 2006-2013) com dados de estudos portugueses prévios publicados recentemente.

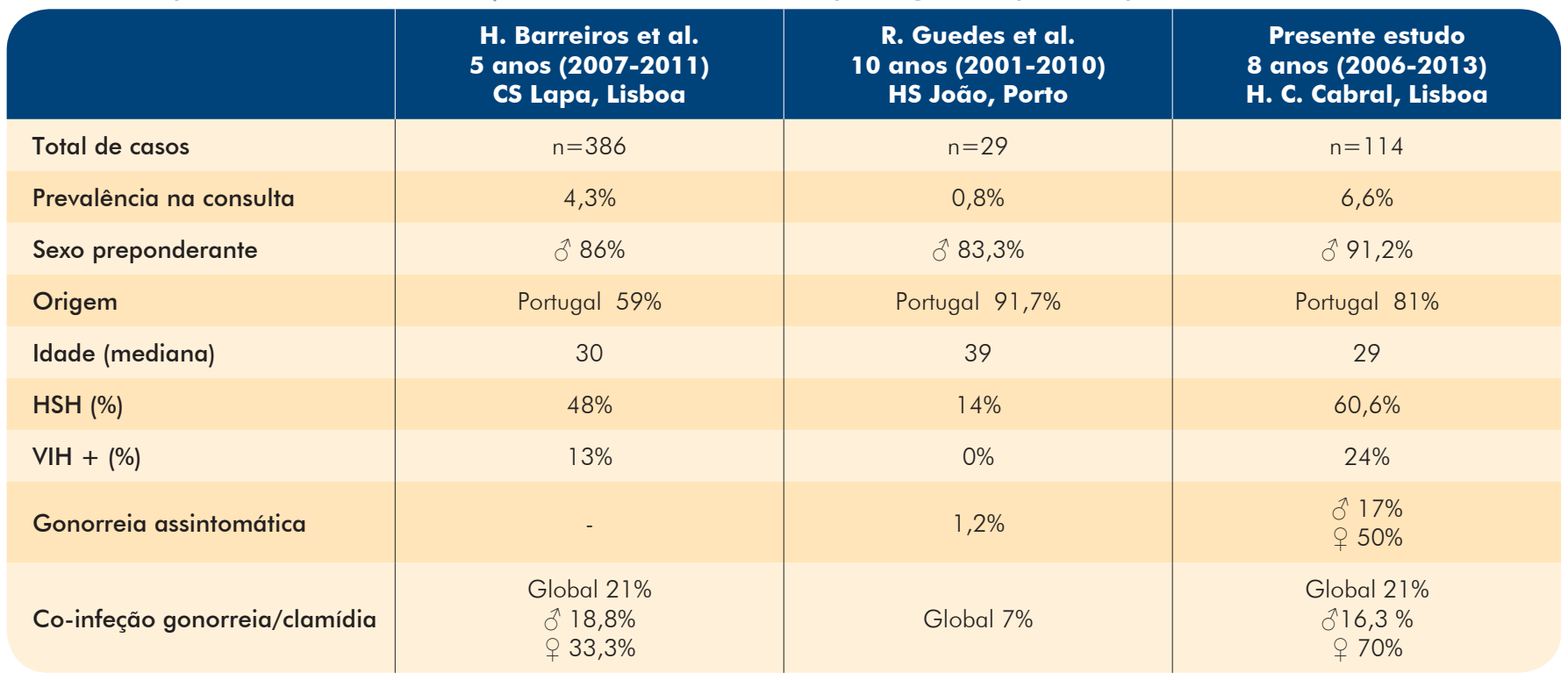


de casos em grupos específicos, nomeadamente os $\mathrm{HSH}$, com $55 \%$ da totalidade dos casos, e os doentes seropositivos para o $\mathrm{VIH}$ que constituem $24 \%$ dos casos. A preponderância do sexo masculino nos diagnosticados com gonorreia é semelhante aos dados publicados por Barreiros e colaboradores e Guedes e colaboradores $^{13,14}$; contudo, a análise da Tabela 3 permite verificar que a percentagem de $\mathrm{HSH}$ com gonorreia é significativamente mais elevada nas populações estudadas no Hospital de Curry Cabral e no Centro de Saúde da Lapa quando comparadas com a população estudada no Hospital de São João no Porto. De igual forma, os diagnósticos de gonorreia em doentes seropositivos para o $\mathrm{VIH}$ é substancialmente superior que nos referidos estudos nacionais (Tabela 3). Estes dados podem ser explicados pelo facto de se tratar de uma Consulta de IST "aberta" e pela forte colaboração desta Consulta com o Serviço de Doenças Infecciosas do Hospital de Curry Cabral.

O número significativo de co-infecções com Chlamydia trachomatis $(21 \%$ na globalidade dos casos e $70 \%$ dos casos no sexo feminino) está de acordo com os dados da literatura. Estes dados corroboram a relevância das técnicas laboratoriais de biologia molecular que permitam a pesquisa concomitante dos dois agentes na mesma amostra bem como a escolha de esquemas terapêuticos eficazes simultaneamente para N. gonorrhoeae como C. trachomatis. ${ }^{11}$

O paradigma da abordagem das infecções sexualmente transmissíveis baseia-se não apenas no diagnóstico e tratamento dos doentes sintomáticos mas também dos contactantes e dos portadores assintomáticos. No presente estudo, $50 \%$ das mulheres com o diagnóstico de gonorreia encontravam-se assintomáticas para esta infecção (todos os diagnósticos foram em localização uro-genital e a PCR não foi realizada em outras localizações). Quanto aos homens, 17\% dos diagnósticos ocorreram em localizações assintomáticas, salientando-se 11 casos de gonorreia assintomática oro-faríngea e ano-rectal em HSH. Koediik e colaboradores ${ }^{15}$ publicaram dados recentemente reforçando a importância de testar as localizações extra-genitais tanto para $\mathrm{C}$. trachomatis como para $\mathrm{N}$. gonorrhoeae, particularmente em grupos de alto risco como os HSH. É notório que o número de casos extra- genitais assintomáticos do presente estudo é limitado, dado que na Consulta de IST do HCC o rastreio desta infecção em localizações extra-genitais em HSH começou apenas em 2013. São necessários mais estudos com rastreio sistemático nas três localizações, nacionais e internacionais.
Quanto à elevada prevalência de gonorreia e outras IST em doentes seropositivos para o $\mathrm{VIH}$, este é um fenómeno preocupante e tendencialmente crescente nos países ocidentais. O fenómeno chamado "HIV prevention fadigue" ("fadiga da prevenção da infecção pelo $\mathrm{VIH}^{\prime \prime}$ ) aparentemente associado a um optimismo excessivo relativamente à terapêutica anti-retroviral e a uma menor preocupação com a transmissão do vírus em doentes sob terapêutica, é provavelmente responsável pelo aumento de prevalência de IST neste grupo específico. ${ }^{16,17}$ A procura de parceiros sexuais nas redes sociais é também um fenómeno recente mas instalado. Margolis e colaboradores procuraram estudar os hábitos dos $\mathrm{HSH}$ seropositivos para o $\mathrm{VIH}$ utilizadores de um sítio da internet para procura de parceiros sexuais; concluíram que 1/3 dos HSH seropositivos para - $\mathrm{VIH}$ admite nos 60 dias precedentes sexo anal sem preservativo com parceiros seronegativos ou de status serológico desconhecido. ${ }^{18}$ Assim, parece evidente a necessidade de delinear estratégias de prevenção que alcancem a população em geral e os subgrupos de maior risco em particular, acompanhando a evolução dos hábitos sexuais.

Numa era de infecção crescente por estirpes de $\mathrm{N}$. gonorrhoeae resistentes aos antibióticos, a importância da notificação e do envio para cultura/teste de sensibilidade aos antimicrobianos do exsudado uretral/endocervical não deve ser descurada. A caracterização das estirpes de N. gonorrhoeae e a detecção de resistências é fundamental para um melhor controlo desta infecção no futuro e cabe aos clínicos, no dia-a-dia, dar o primeiro passo para que este objectivo seja alcançado.

\section{BIBLIOGRAFIA}

1. Ison C. Biology of Neisseria gonorrhoeae and the clinical picture of infection. In: Gross G, Tyring $S$, editors. Sexually transmitted infections and sexually transmitted diseases. Berlin: Springer-Verlag; 2011. p. 77-90.

2. Mehrany K, Kist J, O'Connor W, DiCaudo D. Disseminated gonococcemia. Int J Dermatol. 2003; 42:208-9.

3. European Centre for Disease Prevention and Control (ECDC). Sexually transmitted infections in Europe 1990-2010. [Internet]. Stockholm; 2012. [consultado Jan 2015] Disponível em: http://www.ecdc. europa.eu/en/publications/Publications/201206-Sexually-transmitted-Infections-Europe-2010.pdf.

4. Health Protection Agency (HPA). Sexually transmitted 
infections in men who have sex with men in the UK: 2011 report. London: HPA; 2011.

5. Health Protection Agency (HPA). New data show sexually transmitted infection diagnoses on the rise in England. London:HDP; 2012.

6. Savage E, Marsh K, Duffell S, Ison C, Zaman A, Hughes G. Rapid increase in gonorrhoea and syphilis diagnoses in England in 2011. Euro Surveill. 2012; 17:1-4.

7. Trienekens S, Koedijk F, van den Broek I. Sexually transmitted infections, including HIV, in the Netherlands in 2011 . Bilthoven: Rijksinstituut voor Volksgezondheid en Milieu; 2012.

8. Velicko I, Arneborn M, Blaxhult A. Syphilis epidemiology in Sweden: Re-emergence since 2000 primarily due to spread among men who have sex with men. Euro Surveill. 2008;13:1-5.

9. Velicko I, Unemo M. Increase in reported gonorrhoea cases in Sweden, 2001-2008. Euro Surveill. $2009 ; 14$.

10. Direcção Geral da Saúde (DGS). Doenças de Declaração Obrigatória 2009-2012. Lisboa: DGS; 2014.

11. Bignell C, Unemo M. European Guideline on the Diagnosis and Treatment of Gonorrhoeae in Adults. Int J STD AIDS. 2013; 24:85-92.

12. Tavares E, Fernandes C, Borrego M, Rodrigues A, Cardoso J. Resistência aos antibióticos em Neisseria gonorrhoeae - passado, presente e futuro. Rev Soc Port Dermatol Venereol. 2012; 70:483-93.

13. Barreiros H, Azevedo J, Santo I. Evolução da infecção por Neisseria gonorrhoeae numa população da Consulta de DST do Centro de Saúde da Lapa de 2007 a 2011 . Rev Soc Port Dermatol Venereol. 2011; 7:65-70.

14. Guedes R, Sobrinho-Simões J, Azevedo F, Lisboa C. Infecção por Chlamydia trachomatis e Neisseria gonorrhoeae em utentes de uma consulta de Doenças de Transmissão Sexual - análise de dez anos. Rev Soc Port Dermatol Venereol. 2012; 70:91-7.

15. Koedijk F, Van-Bergen J, Dukers-Muijrers N, Van-Leeuwen A, Hoebe C, Van-der-Sande M. The value of testing multiple anatomic sites for gonorrhea and chlamydia in sexually transmited infection centres in the Netherlands, 2006-2010. Int J STD AIDS. 2012; 23:626-38.

16. Dukers N, Goudsmit J, de Wit J, Prins M, Weverling $G$, Coutinho R. Sexual risk behaviour relates to the virological and immunological improvements during highly active antiretroviral therapy in HIV-1 infection. AIDS. 2001; 15:369-78.

17. Van de Ven P, Prestage G, Crawford J, Grulich A, Kippax S. Sexual risk behaviour increases and is associted with HIV optimism among HIV-negative and HIV-positive gay men in Sydney over the 4 year period to February 2000. AIDS. 2000; 14:2951-3.

18. Margolis AD, Joseph $H$, Hirshfield $S$, Chiasson MA, Belcher L, Purcell DW. Anal Intercourse Without Condoms Among HIV-Positive Men Who Have Sex With Men Recruited From a Sexual Networking Web site, United States. 2014; 41:749-55. 